\title{
La psychothérapie comme logique de production de l'individu : lorsque le social est au coeur même d'un « voyage au fond de soi "
}

\author{
Psychotherapy as a Logic of Production of the Individual: When \\ the "Social " is at the Very Hearth of a "Trip into the Innermost \\ Self"
}

\section{Roch HURTUBISE}

Volume 17, numéro 1, avril 1985

Santé mentale et processus sociaux

URI : https://id.erudit.org/iderudit/001358ar

DOI : https://doi.org/10.7202/001358ar

Aller au sommaire du numéro

\section{Éditeur(s)}

Les Presses de l'Université de Montréal

\section{ISSN}

0038-030X (imprimé)

1492-1375 (numérique)

\section{Découvrir la revue}

Citer cet article

HURTUBISE, R. (1985). La psychothérapie comme logique de production de l'individu : lorsque le social est au coeur même d'un "voyage au fond de soi ". Sociologie et sociétés, 17(1), 73-82. https://doi.org/10.7202/001358ar

\section{Résumé de l'article}

L'analyse présentée dans cet article consiste en une interrogation sur le "caractère" social de la psychothérapie. Des éléments permettant partiellement d'établir ce dernier ont été dégagés de 25 entrevues auprès de thérapeutes. Deux grands ensembles ont ainsi été constitués. Le premier peut être lié aux différentes théories qui soutiennent que la psychothérapie est le "produit d'une société"; au fondement de cette dernière on retrace une logique de production qui se formule dans les termes de travail, d'offre et de demande. Le deuxième ensemble, met en relief la nature du "produit" réalisé dans le cadre de cette relation; c'est une catégorie qui permet d'effectuer un découpage du réel qui constitue l'élément autour duquel la situation peut être construite: l'individu. Cet ensemble peut être associé aux théories qui soutiennent que la psychothérapie est un mécanisme de production de la société. Cette analyse permet d'amorcer, en conclusion, une réflexion sur l'enracinement de la psychothérapie dans un système de rapports sociaux et sur les problèmes théoriques et méthodologiques que soulève l'analyse de ce phénomène du point de vue de ses qualités sociales.
Tous droits réservés @ Les Presses de l'Université de Montréal, 1985

Ce document est protégé par la loi sur le droit d'auteur. L’utilisation des services d'Érudit (y compris la reproduction) est assujettie à sa politique d'utilisation que vous pouvez consulter en ligne.

https://apropos.erudit.org/fr/usagers/politique-dutilisation/ 


\section{La psychothérapie comme logique de production de l'individu: lorsque le social est au cœur même d'un «voyage au fond de soi»}

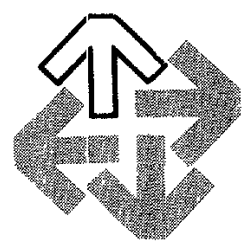

ROCH HURTUBISE

Qu'est-ce qui se passe dans le cadre d'une psychothérapie? Comment analyser cette relation «intime» et «personnelle» dont on dit souvent qu'elle suppose un certain recul à l'égard du social, qu'elle est une expérience en marge de la société? Cette dernière repose-t-elle uniquement sur une logique rationnelle et scientifique? Constitue-t-elle un univers ou seul le «psychologique» règne? N'esi-elle pas aussi relative à des sociétés et des époques données? Telle est du moins l'une des conclusions qu'on peut tirer des débats en sciences sociales sur ce sujet ${ }^{1}$ : c'est alors le contexte social de la psychothérapie qui est mis de l'avant. Une première définition de ce phénomène comme objet de recherche a ainsi été élaborée pour qu'une analyse du point de vue de ses qualités dites «sociales» puisse être faite.

De ces débats, deux tendances peuvent être dégagées. La première met l'emphase sur les «facteurs sociaux» qui influencent cette relation. Elle varie suivant 1. la formation académique, les caractéristiques personnelles, les expériences antérieures, l'âge, l'origine sociale du thérapeute et du patient; 2 . les dimensions organisationnelles et les contraintes structurelles (environnement de travail, conditions de pratique, structure hospitalière $)^{2}$. Dans cette perspective, la relativité au contexte social repose sur le fait que la société «influence» ou «détermine» la nature même de la psychothérapie.

La deuxième tendance situe cette relation dans le cadre plus général d'une analyse du pouvoir et de: la domination. Plusieurs auteurs ont souligné le caractère «politique» de l'intervention en psychiatrie, proposant une analyse de sa fonction sociale. Elle consisterait à assurer le maintien

* Cet article s'inspire largement de mon mémoire de maîtrise les Équipes multidisciplinaires comme objet de recherche en sciences humaines, Département de sociologie, Université de Montréal, 1982. Je remercie le ministère de la Santé et du Bien-ìtre du Canada pour le financement qu'il m'a accordé. Je tiens aussi à remercier les thérapeutes pour leur participation.

1. La psychothérapie fait l'objet de multiples controverses en regard de son utilité et de son efficacité. Szasz, par exemple, soutient que le caractère scientifique de la psychothérapie est un leurre, car il s'agit de la transposition d'une procéfure d'investigation et d'intervention propre à la médecine et aux maladies physiologiques. La psychothérapie serait, selon cet auteur, un mythe, une vague métaphore plutôt qu'une technique rationnelle d'intervention; sa seule réalité palpable consisterait en un type particulier de conversation entre des individus. Cet exemple illustre la nature éminemment critique et politique du débat auquel ont largement participé les sociologues ces dernières années. Voir Thomas Szasz, The Myth of Psychotherapy, New York, Anchor Press, 1978. Toutefois, ce problème de l'utilité est insoluble, la psychothérapie étant à la fols efficace et inefficace selon le point de vue duquel on la regarde. Sur ce thème, voir la revue de littérature dans l'article de Jérôme Guay, «La psychothérapie est-elle efficace», in Psychothérapies Attention, Québec, Québec Science, 1983.

2. Voir la revue de littérature de George J. Allen, Understanding Psychotherapy: comparative perspective. Illinois, Research Press Co., 1977 
de la norme, de la cohésion d'une société - étant donc une forme particulière de contrôle social ${ }^{3}$. Cette fois ce n'est pas en tant que produit d'un ensemble d'influences qu'on explique la relation thérapeutique; elle est partie intégrante d'un système de rapports sociaux.

Ces tendances réfèrent à deux types d'explication, à deux façons d'établir la nature sociale de la psychothérapie. D'une part le social en «amont» de ce phénomène: soit toutes les composantes sociales préexistantes qui, d'une quelconque façon, peuvent être liées à la psychothérapie. L'on soutient alors que la psychothérapie est socialement construite et qu'il est donc possible de l'expliquer par le «social». D'autre part le social en «aval» de la psychothérapie, soit toutes les dimensions du social qui sont médiatisées par celle-ci. Serait mis en relief un autre type de qualités sociales, soit le fait que la psychothérapie est l'un des mécanismes de production de la société; c'est un des lieux où s'effectue la construction de la réalité sociale. Cette fois c'est en quelque sorte la psychothérapie qui permet d'expliquer certains aspects du social.

Si la psychothérapie est relative à l'état de développement de la société, ce n'est donc pas d'une manière singulière et univoque. Définir les qualités sociales de la psychothérapie, c'est mettre en relief la dynamique complexe du social au sein même de cette dernière, le jeu subtil des relations sociales qui se jumèlent et s'enchevêtrent. Autrement dit, il existe plusieurs «entrées» qui permettent de construire la psychothérapie d'un point de vue sociologique ${ }^{4}$.

Dans cet article je veux présenter quelques pistes qui visent à prolonger ce travail de construction sociologique des psychothérapies et qui sont imprégnées des deux types d'explication précédemment identifiés. Ma démarche est inductive et largement exploratoire.

L'analyse des psychothérapies présuppose une certaine délimitation qui permet de saisir «ce à quoi elles correspondent dans la réalité». C'est là une tâche complexe, car plusieurs situations peuvent être regroupées sous le même vocable: du contact familial à l'échange professionnel clinique en passant par les multiples groupes de croissance «semi» professionnels ${ }^{5}$. Je privilégierai l'analyse de psychothérapies dont on pourrait dire qu'elles sont de type «classique», au sens où elles impliquent une relation entre un thérapeute et un patient en milieu professionnel ${ }^{6}$.

J'ai effectué des entrevues auprès de deux groupes de thérapeutes professionnels ( 25 au total) œuvrant dans des institutions du secteur public ${ }^{7}$. Une stratégie d'interview s'inspirant des techniques de stimulation fut adoptée, dans le but dirais-je de reproduire «artificiellement» une psychothérapie ${ }^{8}$. L'objectif de ces entrevues était de reconstituer les activités des thérapeutes comme si ces derniers étaient dans leur situation effective de travail. À cette fin, trois scénarios hypothétiques (histoires de cas) leur ont été soumis ${ }^{9}$. Ces entrevues ouvertes comportaient un minimum de questions, la

3. Sur ce thème voir notamment Robert Castel, le Psychanalysme, Paris, Flammarion, 1981 et Martin L. Gross, les Psychocrates, Paris, Robert Laffont, 1979.

4. Mon objectif n'est cependant pas de substituer une définition sociologique à une définition psychologique du phénomène: la psychothérapie n'a pas que des «qualités sociales». Elle est un objet de recherche susceptible d'être construit de plusieurs points de vue (psychologique, sociologique, politique, etc.)

5. En fait, la relation thérapentique ainsi désignée ne caractérise plus uniquement le rapport entre un thérapeute professionnel et un client; par exemple certains aspects des relations amoureuses sont qualifiés de «thérapeutiques» à cause du bien être qu'elles procurent aux individus. De plus, les thérapies professionnelles sont nombreuses et variées; plus de 250 ont été répertoriées. Richie Herink, The Psychotherapy Handbook, Meridian Books, 1980.

6. Cette délimitation ne doit pas être perçue comme une réduction du phénomène à sa seule dimension professionnelle et institutionnelle. Si une panoplie de situations ont un caractère "psychothérapeutiques», elles ne sont pas pour autant des psychothérapies. Le choix des psychothérapies de type «classique» s'explique donc par le fait qu'elles constituent un modèle par excellence, une relation que l'on pourrait pratiquement qualifier d' «idéal-type», d'où leur intérêt particulier d'un point de vue méthodologique.

7. J'ai réalisé ces entrevues pendant les mois de mai et de juin 1982. La durée de chacune des entrevues varie entre 45 et 90 minutes. Je souligne que les deux groupes de thérapeutes travaillent dans des hôpitaux différents.

8. L'observation directe de la relation thérapeutique pose des problèmes méthodologiques et éthiques. Pour que ce genre d'observation soit possible il aurait fallu obtenir l'autorisation d'une série de comités d'éthique (hôpital, ministère, université) et l'accord des patients. De plus, l'avantage d'une observation directe est sérieusement hypothéquée par le biais introduit par ma présence à titre d'observateur.

9. Cette tâche correspond à une activité spécifique du travail des thérapeutes: l'entrevue d' «intake» qui consiste à rencontrer le patient une première fois afin de discuter de sa situation. Les cas hypothétiques répondent aux critères suivants: a. l'histoire doit être plausible pour les thérapeutes; $b$. I'histoire doit être similaire aux demandes que les thérapeutes reçoivent habituellement; c. l'histoire ne doit pas présenter des références socio-économiques qui entraineraient la non-vraisemblance du cas pour le secteur; $d$. l'histoire ne doit pas entraîner de références systématiques à une profession (par exemple une demande d'assistance-sociale ou de prescription de médicaments). 
volonté étant de saisir la façon dont les thérapeutes formulent le problème et les modalités selon lesquelles ils réagissent aux cas ${ }^{10}$.

Les discours ainsi recueillis sont pertinents à ma démarche d'analyse car ils constituent une expression spécifique de la relation thérapeutique au niveau de la pratique quotidienne des thérapeutes ${ }^{\prime \prime}$. En discutant de tels cas, semblables en bien des points à ceux qu'individuellement ils rencontrent quotidiennement, ils font part de la «connaissance» qu'ils produisent des cas qui leurs sont soumis; construction qui est fondée sur l'explication qu'ils se donnent de leur activité de thérapeute, de la maladie mentale, de leur milieu de travail, de l'intervention, etc. ${ }^{12}$

Quelle mise en situation présuppose une psychothérapie? Comment le thérapeute et le patient participent-ils à cette dynamique? Quels sont les fondements de cette relation? Sur quel type de construction de la réalité repose-t-elle? Comment parle-t-on de cette relation? Quels critères utiliset-on pour la décrire? pour la définir? pour agir? Que cherche-t-on à comprendre? Quelle construction de la situation élabore-t-on? Quel découpage du réel opère-t-on? Voilà le type de questions qui ont guidé la lecture que j'ai faite du matériau.

Je précise que le travail d'analyse ne consiste pas en l'application au matériau d'une grille construite à priori. Il consiste à dégager la psychothérapie — qui constitue l'objet réel de la présente démarche - telle qu'elle est construite au sein de ce matériau; à saisir les modalités spécifiques de construction de l'objet, la grille inhérente à ces discours ${ }^{13}$. Ce ne sont pas les détails, la singularité de chacune des réactions émises qui sont privilégiés. L'objectif est de mettre en relief le «général», les «constantes» qui bien qu'elles se traduisent selon des modalités différentes dans chaque cas, constituent des caractéristiques communes à l'ensemble.

Sur la base des multiples lectures effectuées, il est apparu que les différentes informations contenues dans le matériau pouvaient être groupées. Non pas que la réaction des thérapeutes soit identique en dépit des différences au plan des caractéristiques professionnelles et de l'environnement de travail ${ }^{14}$ ! Mais il est possible de dégager des éléments communs à tous, les thérapeutes faisant intervenir ce que l'on pourrait appeler des «paramètres» ou des «règles» de construction similaires ${ }^{15}$. Deux. grands ensembles ont pu ainsi être constitués. Le premier réfère en quelque sorte au social en «amont», soit à une logique de fonctionnement qui semble fonder un ensemble de rapports sociaux et qui médiatise aussi la psychothérapie. Le deuxième concerne le social en «aval», le résultat même de la psychothérapie, soit la constitution d'une connaissance, la production d'un sens sur une situation donnée.

\section{LA PSYCHOTHÉRAPIE: UNE CERTAINE LOGIQUE DE PRODUCTION}

La psychothérapie constitue une catégorie passablement large, pouvant caractériser des formes d'intervention fort différentes. Toutefois, il semble que quelle que soit la singularité de la psychothérapie

10. Les questions suivantes ont été posées aux thérapeutes: Quel est ou quels sont le(s) problème(s) que vous identifiez? Quel est ou quels sont le ou les aspects sur lesquels il faudrait intervenir? Quel est ou quels sont le ou les moyens à privilégier pour faire cette intervention? Si vous jugez nécessaire de connaître plus d'informations sur ce cas, quelles sont ces informations?

11. L'on pourrait me rétorquer que privilégiant cette approche je recueille uniquement l'opinion des thérapeutes sur ce qu'est la psychothérapie. Certes, sur la base du matériau constitué, une analyse de l'opinion des thérapeutes pourrait être effectuée. Ce n'est cependant pas «la seule» analyse possible, car l'on peut aussi s'intéresser à la psychothérapie en faisant l'analyse de ce que les thérapeutes en disent: ce qui est alors mis en relief c'est une construction de cet objet, qui bien qu'effectué par les thérapeutes ne leur est cependant pas nécessairement exclusive. L'analyse d'interview auprès de «patients> pourrait mettre en relief le fait qu'une certaine construction de la relation est commune aux deux parties.

12. Compte tenu de la nature même des entrevues - où je demandais aux thérapeutes de donner leurs réactions - les informations dont je dispose ne constituent pas des réponses ou des indications précises et parfaitement articulées les unes par rapport aux autres. L'on peut qualifier celles-ci de «premières impressions» des thérapeutes. Ceci explique le fait que les informations disponibles prennent la forme d'hypothèses et sont empreintes de «peut-être».

13. La démarche privilégiée dans le cadre de cette étude s'associe à une certaine approche de l'analyse de contenu. Sur cıtte question je réfêre le lecteur aux textes suivants, l'objet de cet article n'étant pas d'expliquer cette démarche dans ses moindres détails. Jean-Claude Gardin, les Analyses du discours, Neuchâtel, Delachaux/Niestle, 1974; Gilles Houle, «L'idéologie: un mode de connaissance», in Sociologie et sociézés, vol. 11, n 1, 1979; Nicole Ramognino et M. KantoKlein, «Les faits sociaux sont pourvus de sens", in Connexions, vol. 11, 1974.

14. Á cet effet, je souligne que les différences observées sont de l'ordre d'un clivage selon le milieu de travail (un hôpitial par rapport à l'autre) et non pas selon les professions respectives des thérapeutes. Les thérapeutes ouvrant dans un hôpital tendent à avoir des réactions similaires.

15. Les réactions des thérapeutes ne seront donc pas ici considérées en fonction de leur justesse et de leur pertinence en regard des problèmes qui leurs ont été soumis. En fait il n'y a pas de «réaction idéale» ou de «bonne réponse», les situations pouvant donner lieu à des interprétations distinctes relatives à des perspectives théoriques et thérapeutiques, qui bien qu'étant différentes ne sont pas pour autant contradictoires. 
proposée (par exemple le fait qu'elle s'inspire de l'approche analytique ou behaviorale), une certaine façon de «faire», de poser les problèmes est commune.

Dans les entrevues j'ai noté l'absence de comportements thérapeutiques définis explicitement comme étant modulés suivant une relation patient-thérapeute «traditionnelle»; les interviewés ne définissent pas l'intervention en termes de «soins»; les individus des histoires de cas en termes de «malades»; les problèmes qu'ils identifient comme relevant de «pathologies physiologiques». Alors comment se posent-ils?

Généralement, les thérapeutes parlent de «gens», «d'individus». Par-delà des détails distincts, c'est en terme de «travail» qu'ils définissent cette relation. Cependant, ce n'est pas un travail «sur» l'individu, c'est un travail «avec» l'individu ${ }^{16}$. Contrairement à l'acte de soigner - qui implique une relation à sens unique où le thérapeute agit (soigne, traite, guérit) le patient - le travail suppose une action «conjointe», une «collaboration» entre le thérapeute et l'individu. Le potentiel thérapeutique de ce dernier, sa capacité d'être partie agissante est établie.

Je me sentirais confortable pour travailler avec ${ }^{17}$ ce gars là (Groupe A) ${ }^{18}$

Ce n'est pas quelqu'un avec qui je travaillerais. Compte tenu de ses attentes, je ne me sens pas familière (Groupe B).

Le «travail»semble donc constituer un élément définiteur de la relation construite dans le cadre d'une psychothérapie.

Aux dires des interviewés, lorsqu'ils entrent en rapport avec un individu, c'est parce que ce dernier en a fait la demande. Cette notion de «demande» ainsi que celle d' «attente» sont présentes dans la majorité des entrevues. Elles en constituent par ce fait une composante fondamentale étant l'une des «constantes» identifiées au sein du matériau.

Je comprends mal sa demande. À quoi il s'attend? Il y a beaucoup d'éléments intéressants (dans l'histoire) pour voir sa personnalité, ses intérêts, son vécu intérieur. Est-ce qu'il veut de l'aide? Il faudrait préciser la demande car les interventions dépendent de la demande qu'il fait (Groupe B).

Il n'y a pas de demande qui soit formulée. Il faudrait savoir ce qu'il souhaite sinon, il risque de ne pas s'impliquer, il n'y a pas assez d'informations pour savoir s'il est apte à une psychothérapie. Est-ce qu'il a vraiment mal? II faudrait lui demander comment on peut ]'aider, qu'est-ce qu'il veut changer, qu'est ce qu'il voudrait qu'on lui offre? (Groupe B). Je n'accepterais pas facilement ce cas en thérapie. Il risque une déception face à la réalité d'une thérapie et semble avoir des attentes magiques. Je ferais bien préciser les attentes (Groupe A).

On note d'ailleurs qu'à ce processus d'identification de la demande correspond celui de l'élaboration d'une réponse qui, dans les termes des thérapeutes, se formule comme offre.

Qu'est ce qu'il voudrait qu'on lui offre? (Groupe A).

On pourrait lui offrir une thérapie de compréhension dynamique... d'inspiration psychanalytique (Groupe B).

Je lui offrirais ce qu'elle demande: une psychothérapie... à orientation analytique. Dans la mesure où il y a une demande, la réponse est analytique (Groupe B).

Cette évaluation de l'offre et de la demande est d'ailleurs une procédure complexe, qui nécessite une analyse minutieuse de la situation:

Il a l'air d'avoir un peu une idée magique de ce qu'est une psychothérapie. J'aurais tendance à lui expliquer un peu ce qui existe... ce qui peut être fait, dans quel sens ça va... pour qu'il puisse clarifier sa demande. Je lui dirais qu'on connait des personnes qui peuvent l'aider. Je lui donnerais une liste de psychologues en lui disant qu'il peut toujours rappeler $\mathrm{s}$ 'il a des problèmes (Groupe $\mathrm{A}$ ).

Je ne ferais pas de proposition... d'abord il faut établir une relation de confiance avec lui, laisser le temps à la relation de s'établir. Il est important de ne pas reproduire les comportements des parents... donc il faut faire l'investigation des demandes... prendre le temps d'établir une relation de confiance même si ça prend du temps (Groupe B).

16. Je précise que si l'on travaille «avec» l'individu, on ne travaille cependant pas «sur» l'individu en tant que totalité. L'intervention, bref le travail, suppose un découpage de ce dernier (comme nous le verrons ultérieurement) dont le résultat (corps, agressivité, etc.) devient l'objet même du travail. Par exemple «on travaille avec les images qu'elle a de la peur».

17. C'est moi qui souligne.

18. Les informateurs sont ici identifiés en fonction du groupe auquel ils appartiennent; chacun de ces groupes correspondant à un hôpital donné. 
Ce processus d'offre et de demande constitue un élément clé, définiteur et organisateur du matériau, et de la façon dont au sein de ce matériau, la psychothérapie est construite ${ }^{19}$. Se trouve donc définie, une organisation du thérapeute et du patient dans un contexte particulier, celui d'une psychothérapie. Autrement dit, les notions d'offre et de demande semblent pertinentes aux thérapeutes car elles permettent de faire un découpage «opérationnel» de la réalité qui est l'objet de leur attention; cette première mise en forme constitue en quelque sorte un travail préalable à la production d'une explication.

\section{LA CONNAISSANCE PRODUITE: UNE MISE EN FORME SELON DES CATÉ- GORIES DONNÉES}

Dans la section précédente, la psychothérapie est apparue comme relevant d'une logique de fonctionnement, d'une certaine façon de formuler les rapports entre des personnes. Ces aspects réfèrent à une logique de production. Le deuxième ensemble d'informations contenues dans le matériau concerne le produit de cette relation: une connaissance de l'individu ${ }^{20}$. En fait, il est apparu que certaines organisations sont récurrentes, que certains découpages sont systématiquement utilisés: seraient mises en relief ce que l'on pourrait appeler les «catégories» d'un système sémantique au fondement de la psychothérapie ${ }^{21}$.

Les thérapeutes proposent donc un certain ordre logique d'organisation des informations afin de les resituer dans une «problématisation» de la situation. Si le réel se présente de façon chaotique, les thérapeutes possèdent une «grille de lecture» leur permettant d'organiser ces informations anarchiques sous la forme d'un savoir relativement clair et logique. Finalement le projet d'une psychothérapie est d'élaborer une explication de l'individu dans une situation donnée. Ce travail semble d'abord consister à cerner de façon précise «ce dont on parle», à produire une définition de l'objet à expliquer.

Tous les éléments convergent: ce sont les différents processus par lesquels sont établies les «limites», les «frontières» de l'individu. Ce qui est ainsi construit ce sont des «catégories» en fonction desquelles il est possible de le «penser». La psychothérapie pourrait être définie comme un mécanisme qui permet une mise en forme de l'individu. Cette connaissance serait donc la constitution d'une catégorie spécifique qui permet d'organiser et de donner sens au réel: l'individu.

\subsection{L'INDIVIDU PORTEUR DE CARACTÈRES}

L'individu est décrit en fonction des caractéristiques qui lui seraient intrinsèques, de qualités qui permettent de le cerner comme une entité «finie» et «organisée». Ce découpage est notamment celui du conscient et de l'inconscient:

J'aime mieux travailler avec le conscient, plutôt qu'avec l'inconscient. Je ne tiens pas compte du rêve même si c'est évident que c'est lui qui est sur l'île et qu'il se sent bombardé (Groupe A).

De «l'imaginaire» de ce dernier;

Je serais curieuse de voir si sa vie phantasmatique est riche (Groupe B).

Il est capable d'activité onirique donc accessible à un processus thérapeutique (Groupe B).

19. Il s'agit là d'un rapport d'offre et de demande, d'une logique dont les termes ne peuvent être définis indépendamment l'un de l'autre. La demande et l'offre s'interpellent, elles ne sont donc pas respectivement spécifiques aux thérapeutes et aux patients. La demande ce n'est pas ce que l'individu présente (son malaise, son problème, son histoire): c'est la construction de cet individu et de ce qu'il présente du point de vue d'une relation thérapeutique en voie de définition. L'offre ce n'est pas ce que le thérapeute propose: c'est la façon dont est construit ce dernier et son environnement de travail du point de vue c' une relation thérapeutique potentielle.

20. À ce sujet, voir les travaux de Berger et de Lévi-Strauss ou la relation thérapeutique est définie comme une activité visant à produire une connaissance sur une situation donnée. Autrement dit, ce qui se passe entre un patient et un thérapeute, c'est la mise en forme d'une certaine expérience d'un point de vue spécifique. C'est donc aussi dans la perspective d'uné sociologie de la connaissance que peut être privilégié l'étude de cette relation au sens où elle constitue l'un des lieux - ayec sa dynamique et ses mécanismes propres - où les individus se forgent une connaissance de leur vie, l'un des moyens par lesquels ils établissent leur rapport au monde et aux autres hommes. Peter L. Berger, «Toward a sociological understanding of psychoanalysis», in Social Research, vol. 32, $\mathbf{n}^{\circ}$ 1, 1965, 26-41 et Claude Lévi-Strauss, «L'efficacité symbolique», in Anthropologie structurale, Plon, Paris, 1958.

21. Les catégories dégagées ne sont pas exhaustives et mutuellement exclusives, elles réfêrent à des principes généraux en fonction desquels une situation est décrite. C'est pourquoi les exemples qui suivent peuvent souvent être associés à plus d'une catégorie. 
De ses émotions, de ses sentiments, et de tout ce qui relève de l'affect.

Au niveau des affects, pas facile à faire à priori. Il ne doit pas être capable d'en parler facilement. J'aimerais le voir, peut-être que moi je ne perçois pas d'affect, alors que peutêtre en le voyant j'aurais l'impression de plus le sentir (Groupe B).

J'aurais tendance à avoir envie d'informations de l'ordre du flair et du pif... À travers son discours, qu'est-ce qui se dégage? L'odeur du bonhomme c'est quoi? (Groupe B).

Ces caractérisations de l'individu permettent au thérapeute de cerner les dimensions pouvant être utilisées dans le cadre d'une psychothérapie.

Ce qu'on voit beaucoup, ce sont des signes au niveau du corps. Le corps est souvent une porte d'entrée... C'est possible de travailler avec ça (Groupe A).

Ces informations sont de même type car elles correspondent à un certain découpage de l'individu dont le cas est discuté. Seraient par là délimitées des zones ou des régions de l'être qui constitueraient des «portes d'entrées» rendant accessibles aux thérapeutes, l'individu et son problème (ses causes et ses fondements). L'on pourrait en quelque sorte parler d'une mise en forme de l'individu dans les termes d'une activité thérapeutique, mise en forme définitrice du champ d'intervention des thérapeutes: le conscient, l'inconscient, le réel, l'imaginaire, l'affect, le corps, etc.

\subsection{UNE CONSTRUCTION DE L'INDIVIDU DANS LE TEMPS}

Les thérapeutes, bien que selon des modalités distinctes, construisent l'individu en référant au «temps», que ce soit pour situer ce dernier dans le passé ou dans le présent.

Il n'y a pas de problème actuel. Je ne sais pas pourquoi elle consulte actuellement (Groupe A).

Dans ma démarche personnelle je travaille plus sur le «ici et maintenant» que sur une analyse du passé (Groupe A).

Il y a toujours moyen de travailler sur le présent pour rejoindre le passé (Groupe B).

C'est le genre de mec qui me dirait: pourquoi je parlerais de ma vie passée, mon problème est dans le présent. Il est difficile de travailler en analyse avec eux tant qu'ils ne sont pas centrés sur eux ... Se comprendre soi-même, ce n'est pas un travail nécessairement facile (Groupe B).

Cette «dimension temps» constitue un élément organisateur de la connaissance produite dans le cadre d'une relation thérapeutique. Les exemples suivants montrent comment, sans qu'il y ait de référence explicite au temps, le discours des thérapeutes - bien que de façon différente selon les perspectives théoriques privilégiées - consiste en une construction du cas à travers une temporalité donnée. Ainsi, dans le cas d'un groupe de thérapeutes, quels que soient les éléments retenus, c'est toujours au présent qu'ils les discutent, c'est dans l'actuel qu'ils racontent l'histoire.

Quelle est l'intensité de la dépression? Pleure-t-il? A-t-il des idées suicidaires? C'est peutêtre purement situationnel: une réaction dépressive normale à la perte de son amie (Groupe A).

C'est un gars qui est à la recherche de ce qu'il veut faire et être. C'est ça son problème (Groupe A).

Pour ce groupe de thérapeutes, les références au passé, aux antécédents familiaux, à l'enfance, aux liens avec les parents pendant cette période, sont pratiquement inexistantes. Lorsqu'ils font intervenir «la famille», c'est sous l'angle des rapports actuels et de la dynamique présente.

Il est proche de l'adolescence. Il se peut qu'il devienne éventuellement plus sociable... Il est jeune, c'est un âge critique pour la psychose, la séparation d'avec les parents peut être l'élément déclencheur (Groupe A).

Le point qui reste et je n'accorde pas beaucoup d'attention, c'est l'histoire familiale. Je ne centre pas mon attention sur le passé ... Il ne me semble pas important de modifier le passé... Je ne pourrais pas changer sa relation avec ses parents (Groupe A).

Cependant, les thérapeutes de l'autre groupe tendent à situer l'histoire dans un passé donné. C'est en général en fonction de ce dernier que l'on interroge le cas.

Il y a beaucoup d'informations qu'on peut demander sur l'histoire de cet enfant. Quels sont ses antécédents? Pourquoi est-il fils unique? Nous ne savons rien de son enfance, de son développement... A-t-il été un enfant désiré par ses parents (Groupe B).

Le concept de diagnostic ne m'intéresse pas... Le problème c'est qu'il faut mettre en évidence l'histoire de l'enfant avant de poser un diagnostic (Groupe B). 
Dans ces cas, les réflexions des thérapeutes relatives à l'histoire familiale, concernent les souvenirs qu'en conserve l'individu, le «ce qui en reste» chez cette personne. Par ces références aux antécédents familiaux, l'on présente aussi dans une certaine perspective, une interprétation, une explication de la situation actuelle des cas. Ainsi si le temps «passé» est celui privilégié pour construire le cas, ces thérapeutes procèdent aussi dans une certaine mesure d'une articulation entre le «passé» et le «présent».

C'est très clair... C'est un bonhomme déprimé depuis longtemps. Il s'est toujours débattu avec cette dépression profonde liée à l' histoire familiale (Groupe B).

C'est la relation au père qui se généralise avec tous les hommes. Elle reproduit l'image de sa mère qui se laissait battre comme s'il fallait qu'elle endure tout (Groupe B).

D'ailleurs le «ce qui reste» du passé chez l'individu semble même constituer dans certains cas un lieu privilégié d'intervention.

C'est un cas pour une psychothérapie d'inspiration analytique axée sur la relation avec les parents... avec les parents il y a beaucoup de choses qui ne sont pas réglées... Il y aurait lieu de travailler au niveau de cette relation (Groupe B).

\subsection{L'INDIVIDU DANS LE MONDE}

Comme les exemples présentés dans les deux sections précédentes le laisse supposer, l'individu n'es: pas seulement construit en fonction de caractéristiques qui lui seraient intrinsèques, mais aussi selon ce qui permet de le distinguer des autres ou de l'extérieur.

\subsubsection{L'INDIVIDU DANS SON ENVIRONNEMENT IMMÉDIAT}

Les thérapeutes définissent l'individu en regard de ce que l'on pourrait appeler son milieu immédiat: sa famille, ses proches, son travail, etc.

Quels sont ses antécédents? Ceux de ses parents? Ceux de son histoire familiale? (Groupe B).

Le problème que j'identifie est un problème d'isolement et d'anxiété qui peut-être relié aussi avec l'histoire familiale... Le fait qu'il coupe avec sa famille peut entrainer un problème d'anxiété, d'ambivalence dans son milieu (Groupe A).

Par rapport à ce que j'identifie comme problème, je pense qu'il ressort beaucoup d'isolement, beaucoup de solitude... Les rapports avec les gens ont l'air difficiles... Il n'a pas beaucoup de support autour de lui, il est tout seul... tout seul dans la ville et il a l'air de se sentir assez seul dans le groupe où il évolue. Même les contacts avec ses parents sont difficiles (Groupe A).

Ainsi, l'histoire de la famille lorsqu'elle est mise en relation avec la situation actuelle devient pour les thérapeutes un élément explicatif du cas.

Comment ça se fait que tout est centré sur les hommes. Elle s'identifie à sa mère et reproduit le même «pattern» (Groupe B).

Certains considèrent la relation avec la famille comme le lieu même de la définition du problème de l'individu concerné, l'objet du regard que posent les thérapeutes.

Le problème premier, c'est la relation avec les parents (Groupe B).

$\mathrm{Ce}$ «milieu immédiat» ne fait pas seulement partie du processus d'interprétation des cas. Pour les thérapeutes, si le problème implique le rapport de l'individu avec ce «milieu», leur intervention se doit de considérer cet aspect.

Je lui proposerais une psychothérapie, pas de médication, sur le problème d'intégration au cegep... Je viserais la ventilation... et favoriserais la socialisation (Groupe A).

Je regarde beaucoup sa relation avec elle même... et par ricochet la façon dont elle se relie aux autres. Ça pourrait l'amener à être plus sérieuse et plus unifiée, à avoir une meilleure image d'elle-même, à se relier mieux avec les autres (Groupe A).

Ainsi, le cas se trouve donc délimité, distingué non seulement par rapport à ses propriétés intrinsèques (conscience, corps, imaginaire) mais aussi par la façon dont on peut l'identifier comme une entité autonome, au sein d'un réseau de relations (famille, travail, école). Car dans cette perspective, si l'on tient compte de l'environnement, ce n'est pas pour définir les problèmes de cet environnement, mais bien pour situer le cas par rapport à cet environnement. Il s'agit donc d'une procédure par laquelle «l'individu» est mis en relief. 


\subsubsection{LINDIVIDU PAR RAPPORT Aं UN CORPUS DE CONNAISSANCE DONNÉ: LA PSYCHIATRIE}

Cette qualification de la spécificité de l'individu est aussi établie en regard de ce que l'on pourrait appeler une mise en situation des cas particuliers au sein du général qu'est l'ensemble des cas types en psychiatrie: psychose, névrose, etc. Le travail des thérapeutes consiste donc à distinguer le cas, à établir sa singularité en regard des problèmes généralement rencontrés.

Je lui poserais des questions sur ses pensées: a-t-il des idées bizarres, des hallucinations, des délires? Ça laisse suspecter une existence schizoïde. Ça fait penser à un problème qui est peut-être plus symptomatique qu'un problème psychotique (Groupe A).

Il faut éliminer un aspect psychotique chez ce monsieur là... Quoique d'après ce qu'on a ici, il ne semble pas qu'on ait des éléments qui militent dans ce sens là (Groupe A).

Moi la seule affaire par rapport à ce cas-là, c'est que je trouve qu'il nous manque des choses importantes. Il veut avoir le contrôle de lui. C'est quoi les moyens qu'il veut prendre pour changer le monde. Moi mon feeling, c'est qu' il y a peut-être des éléments psychotiques dans tout ça (Groupe B).

Cette dame-là, à première vue, présente clairement des symptômes de nature névrotique (Groupe A).

D'ailleurs, cette distinction fonde dans certains cas la décision des thérapeutes de proposer une psychothérapie.

Sûrement qu'elle pourrait être gratifiante en thérapie parce qu'elle n'est pas psychotique (Groupe B).

C'est le cas le plus intéressant. Ce n'est pas psychotique. J'aime les gens qui ont milité dans les mouvements de gauche (Groupe B).

On remarque donc la présence d'une opposition psychotique/non psychotique comme catégories fondant une classification des cas. D'autres couples peuvent être associés à cette procédure: (cas) urgent/non-urgent, (problème) grave/léger, etc. Seraient introduits ici les schèmes de référence issus des savoirs des thérapeutes; ceux-ci permettraient l'induction, le fait de reconnaître les aspects globaux des différents cas et, à leurs mesures, adapter en conséquence la relation thérapeutique à définir.

\subsubsection{L'INDIVIDU PAR RAPPORT Aे UN SYSTĖME DE DISTRIBUTION DE SOINS}

C'est aussi en termes de positionnement que peuvent être comprises les informations concernant les possibilités de référence à l'extérieur ou de prise en charge. Toutefois, l'induction du particulier au général s'élabore ici en fonction d'un autre ensemble: il ne s'agit plus d'une référence aux savoirs des thérapeutes mais à la connaissance qu'ils ont du système de distribution de soins dans toute sa diversité.

Dans le cadre de la clinique on ne le prendrait pas ici, si ce n'est pas important... Ce serait une référence à l'extérieur pour psychothérapie et relaxation en bureau privé. Je lui donne une liste de psychologues et de psychiatres... il choisit parmi un ensemble de thérapeutes (Groupe A).

Dans le cadre d'ici, ça serait une référence à l'extérieur. Si j'étais dans le contexte comme clinicien en pratique privée je le verrais (Groupe A).

C'est dans les termes d'une distinction entre «psychiatre d'ici» et «psychiatrie d'ailleurs» que peut être retraduite la différence entre respectivement, «prendre en charge» et «référer». Cet «ici»et cet «ailleurs» renvoient davantage à des champs d'interventions distincts de la psychiatrie qu'à diverses approches thérapeutiques. Pour reprendre les expressions des thérapeutes, il s'agit du même coup d'une différenciation entre le privé et le public, qui superpose la distinction entre cas non psychotiques et psychotiques.

II ne s'agit pas d'une psychose. Ici à l'hôpital on traite uniquement des psychotiques... ou des gens qui ont des problèmes tellement majeurs que leur vie est handicapée (Groupe A). Nous avons tellement de demandes que l'on doit ménager nos énergies. $L$ 'approche multidisciplinaire, c'est pour les psychotiques. La psychiatrie privée s'occupe des névrotiques... Les gens qui font appel à nous sont souvent référés au privé... Nous gardons plus nos interventions pour la grosse psychiatrie. (Groupe A)

Les thérapeutes évaluent en quelque sorte l'adéquation à établir entre un cas et le type de relation thérapeutique à définir, posant qu'elle diffère suivant l'une ou l'autre des unités de ce sytème de soins. Dans ce cas c'est non seulement le patient qui est positionné dans le système de distribution de soins, c'est aussi le thérapeute. 


\section{CONCLUSION: L'AVAL ET L'AMONT, DEUX DÉCOUPAGES PARTIELS}

Deux grands ensembles d'informations ont pu être dégagés: ils semblent constituer différents angles qui permettent d'établir le caractère social de la psychothérapie. Le premier réfère à une logique de production, à ce que j'ai appelé en introduction le social en «amont». Par-delà les différences entre les thérapeutes, la même dynamique d'offre, de demande et de travail a pu être identifiée. La psychothérapie serait «médiatisée» par une certaine logique qui caractérise plusieurs rapports sociaux. Cette logique d'offre et de demande n'est-elle pas de quelques manières au fondement même des sociétés actuelles, de leur économie capitaliste moderne, de ce que l'on appelle les sociétés de consommation? Ne pourrait-on pas faire l'hypothèse que le travail réalisé suivant cette logique serait le propre de relations thérapeutiques d'autres types (non psychiatriques)?

Sur cette base peut-on déduire une explication dans les termes d'un rapport de causalité? Bien qu'on puisse retracer au niveau même de la psychothérapie une logique de fonctionnement qui est généralement associée aux rapports sociaux dits économiques, on ne peut conclure à une détermination de l'économique sur la relation ici observée. Cette affirmation (dans la perspective où elle pourrait éventuellement être formulée) nécessiterait une analyse plus approfondie. Cependant, il existe au fondement de la psychothérapie une logique qui déborde cet objet spécifique, car elle caractérise d'autres phénomènes au sein de la société. Autrement dit, la psychothérapie «fonctionne» de la même façon que d'autres rapports sociaux.

Dès lors, un ensemble de questions peuvent être soulevées. Quel est le principe de cette logique? Au fondement de quels rapports la retrouve-t-on? Quels sont les mécanismes et les modalités par lesquels, au sein d'une société donnée, s'effectue ce type de problématisation de la réalité?

Le deuxième ensemble réfère aux différents aspects par lesquels l'individu est construit; au fait que la psychothérapie constitue un processus de production d'une catégorie donnée. Elle est donc non seulement socialement construite, elle construit aussi le social car produisant des connaissances ayant socialement valeur de réalité. L'individu est délimité en fonction de caractéristiques qui lui seraient intrinsèques, d'une certaine temporalité, d'un environnement immédiat, d'un système de distribution de soins et d'un savoir. Seraient ainsi dégagés les «paramètres» d'un univers sémantique au fondement de la connaissance de l'individu produite dans le cadre d'une psychothérapie.

Cette connaissance est «complexe» au sens où elle renvoie à plusieurs univers de référence et non pas à un système unique. Cette complexité n'est pas seulement relative au fait que plusieurs aspects du réel sont intégrés. Plusieurs logiques d'interprétation du monde se jumèlent. «Travailler» avec un individu, ce n'est pas uniquement interpréter sa situation en fonction d'un système de catégorisation des caractéristiques psychiques; d'autres éléments interviennent systématiquement au niveau même de la construction élaborée: les contraintes du milieu, les impressions, les visions du monde ${ }^{22}$.

Peut-on affirmer que la catégorie produite est à l'extérieur du social? L'individu est identifié en fonction d'une certaine autonomie, d'une finitude qui ne prennent sens qu'à travers un processus de référence au social. Comme si cette construction appelait inévitablement cet «extérieur». Tout se passe comme si la définition de l'individu ne pouvait être élaborée sans référence au social. En fait, la connaissance produite dans ce cas est essentiellement une connaissance de l'individu dans le monde et non pas de l'individu hors du monde. Par ce deuxième ensemble seraient mis en relief certains des aspects de la psychothérapie qui réfère au social en «aval»; soit le produit même de cette dernière au sein d'un système de rapports sociaux.

Doit-on conclure de cette analyse que la psychothérapie doit-être analysée en fonction du social en «amont» et du social en «aval»? Que c'est de cette façon qu'on peut cerner sa relativité à un contexte social donné? Bien que l'image soit significative, elle ne doit cependant pas être perçue comme un carcan dans lequel il faut figer toute analyse. Son défaut est de laisser croire que le social est «à côté̀ (devant et derrière) du phénomène. Ne devrait-on pas aussi pouvoir conclure que la psychothérapie est «en soi» sociale?

Dans le cas de la rivière, l'amont et l'aval constitue deux découpages de la masse d'eau qui s'écoule dans un lit. Pourtant, la rivière n'est qu'une, n'est que le mouvement de l'eau dans un

22. Emerson et Pollner ont souligné la complexité du processus de prise de décision des thérapeutes. Selon cette étude les facteurs «subjectifs» interviennent tout autant que les facteurs «objectifs» pour déterminer l'urgence d'un problème psychiatrique. Robert M. Emerson et Melvin Pollner, «Policies and practices of psychiatric case selection», in Sociology of Work and Occupations, vol. 5, n" 1 , février 1978. 
espace donné. L'amont et l'aval sont les découpages effectués par un observateur situé en un point donné et jetant un regard sur ce que semble être la totalité de ce point. En fait, la rivière ce n'est pas d'un côté l'amont et de l'autre l'aval; ces derniers constituent les indices qui permettrent d'identifier partiellement la dynamique de l'ensemble.

Ne peut-on pas faire les mêmes observations au niveau de l'analyse. L'amont n'appelle-t-il pas l'aval? Si l'on est en présence d'une certaine logique de production, ne sommes-nous pas aussi inévitablement en présence d'un produit? La psychothérapie n'est-elle pas aussi un mécanisme qui permet de reproduire et de perpétuer la logique d'offre et de demande? N'assure-t-elle pas la persistance de ce type de problématisation de la réalité au sein des sociétés actuelles? Les préoccupations pour l'individu ne constituent-elles pas un mouvement amorcé depuis quelques siècles? Dans cette perspective la psychothérapie ne peut-elle pas être associée aux différents mécanismes qui assurent le processus d'individuation dans les sociétés occidentales modernes ${ }^{23}$ ?

Le social n'est pas derrière et devant la psychothérapie. Il est comme dans le cas de la rivière tout au long, de manière continue à travers un système de rapports sociaux. Le social en «amont» et le social en «aval» constituent donc deux découpages fragmentaires dont l'avantage est l'identification des «indices» qui pourraient éventuellement permettre de saisir la dynamique des rapports sociaux qui traverse la psychothérapie.

\section{RÉSUMÉ}

L'analyse présentée dans cet article consiste en une interrogation sur le «caractère» social de la psychothérapie. Des éléments permettant partiellement d'établir ce dernier ont été dégagés de 25 entrevues auprès de thérapeutes. Deux grands ensembles ont ainsi été constitués. Le premier peut être lié aux différentes théories qui soutiennent que la psychothérapie est le «produit d'une société»; au fondement de cette dernière on retrace une logique de production qui se formule dans les termes de travail, d'offre et de demande. Le deuxième ensemble, met en relief la nature du «produit» réalisé dans le cadre de cette relation; c'est une catégorie qui permet d'effectuer un découpage du réel qui constitue l'élément autour duquel la situation peut être construite: l'individu. Cet ensemble peut être associé aux théories qui soutiennent que la psychothérapie est un mécanisme de production de la société. Cette analyse permet d'amorcer, en conclusion, une réflexion sur l'enracinement de la psychothérapie dans un système de rapports sociaux et sur les problèmes théoriques et méthodologiques que soulève l'analyse de ce phénomène du point de vue de ses qualités sociales.

\section{SUMMARY}

The analysis presented in this paper consists of a questioning of the «character» of psychotherapy. Elements which make it possible to partially establish this character were isolated from twenty-five interviews carried out with therapists. Two main groups of elements were constituted in this way. The first group can be linked to the various theories claiming that psychotherapy is the "product of a society», at the basis of which we can trace a logic of production which can be formulated in terms of work and of supply and demand. The second group throws light on the nature of the «product» achieved in the framework of this relationship; it is a category which makes it possible to isolate from reality that which constitutes the element around which the situation can be constructed - the individual. This group can be associated with the theories claiming that psychotherapy is a mechanism of the production of society. In conclusion, this analysis is the starting point for reflections on the entrenchment of psychotherapy in a system of social relationships and on the theoretical and methodological problems raised by the analysis of this phenomenon from the point of view of its social qualities.

\section{RESUMEN}

El análisis que se presenta en éste articulo consiste en una interrogación sobre el «caracter» social de la psicoterapia. Elementos que permiten parcialmente de establecer este último se han puesto en relieve en 25 entrevistas a terapeutas. Dos grandes conjuntos han sido constituidos. El primero puede estar ligado a las diferentes teorías que sostienen que la psicoterapia es el «producto de una sociedad»; a la base de ésta teoria encontramos una lógica de producción que se formula en los terminos de trabajo, de oferta y demanda. El segundo conjunto, pone en relieve la naturaleza del «producto» realizado en el cuadro de esta relación; es una categoría que permite efectuar un corte de lo real que constituye el eleménto alrededor del cual la situación puede construirse: el individuo. Este conjunto puede ser asociado a teorías que sostienen que la psicoterapia es un mecanismo de producción de la sociedad. Este análisis permite el inicio, en conclusion, de una reflexión sobre el arraigamiento de la psicoterapia en un sistema de relaciones sociales y sobre los problemas teóricos y metodológicos que plantea el análisis de éste fenómeno desde el punto de vista de sus cualidades sociales.

23. La notion d'individu, non pas au sens de sujet empirique, mais en tant qu'entité morale autonome, est l'objet de nombreuses recherches historiques et anthropologiques. Cette catégorie serait un produit propre aux sociétés occidentales modernes. La relativité de la notion d'individu à des contextes sociaux historiquement délimités, peut-être établie sur la base des travaux de plusieurs auteurs. Selon Lévy-Bruhl, les hommes dit primitifs ne possèdent pas de concept différencié de leur «moi»; en fait, ils ne s'appréhendent pas comme différents de l'univers des choses et des symboles qui les entourent. Mauss de son côté, s'est intéressé au développement de cette notion, aux diverses constructions que les hommes en ont élaborées des débuts de la chrétienté à l'ère moderne. À ces travaux peuvent être associés ceux de Foucault qui à travers différents champs (la prison, la folie, la sexualité), est préoccupé par les multiples «problématisations» de l'individu élaborées en Occident. Marcel Mauss, «La notion de personne», in Sociologie et Anthropologie, Paris PUF, 1950; Michel Foucault, Histoire de la sexualité, vol. 2. l'Usage des plaisirs, Paris, Gallimard, 1984. 